\title{
Assessing Conformance to Medication Treatment Guidelines for Schizophrenia in a Community Mental Health Center (CMHC)
}

\author{
Mona Goldman, Ph.D. \\ Daniel J. Healy, M.D. \\ Timothy Florence, M.D. \\ Lorelei Simpson, M.A. \\ Karen K. Milner, M.D.
}

\begin{abstract}
The feasibility of implementing current medication treatment guidelines in a community mental health clinic was examined in this cross-sectional and retrospective study. Specifically, two issues were addressed: 1) could we identify a set of clinical guidelines whose utilization could be monitored with a routine medical chart review; and 2) were the requisite data for such a review available in the medical records. An examination of three sets of published guidelines revealed 5 specific guidelines that could be operationalized and monitored. Records were reviewed for a random sample of two-thirds of all patients currently diagnosed with schizophrenia and schizoaffective disorder $(n=309)$. Information was collected from the medical record on patient demographics, diagnosis, duration of illness, symptoms and side effects, and medication information. Rates of conformance to the 5 guidelines ranged from a high of $97 \%$ to a low of $43 \%$. The use of current guidelines to improve treatment in community clinics appears to be limited by the deficiencies in the medical record and the structure of the guidelines themselves. Standardized progress notes and computerized prescribing
\end{abstract}

Mona Goldman is Research Investigator, Daniel J. Healy is Lecturer, Timothy Florence is Clinical Instructor, Lorelei Simpson is Research Assistant and Karen K. Milner is Clinical Assistant Professor, all in Department of Psychiatry, University of Michigan Health System.

Address correspondence to Karen K. Milner, M.D., University of Michigan Health System, B1D102, UH, 1500 E. Medical Center Drive, Ann Arbor, MI 48109-0020; e-mail: kmilner@ umich.edu.

This study was funded by a grant from the Ethel and James Flinn Family Foundation. The authors also thank Janet Neuhauser, M.A., for her efforts and contributions. 
programs will improve conformance, and permit the rapid and accurate assessment of conformance to guidelines in community clinics.

KEY WORDS: schizophrenia; guideline conformance; CHMC.

\section{INTRODUCTION}

The introduction of the atypical antipsychotics ushered in a new era in the treatment of schizophrenia. They offer a broader spectrum of efficacy with decreased risk for movement disorders and the promise of increased patient compliance (Jibson and Tandon, 1998). However, because the cost of the newer antipsychotics is higher than that of the older conventional antipsychotics (DeQuardo and Tandon, 1998), decisions regarding the allocation of limited resources have to be raised (Doherty, 1999). To ensure that treatment decisions are rational and evidence-based, professional and government agencies have published clinical practice guidelines and algorithms for the treatment of severe mental illnesses (American Psychiatric Association, 1997; Lehman and Steinwachs, 1998a; The Expert Consensus Guideline Series, 1999; Chiles, Miller, Crismon, Rush, Krasnoff, and Shon, 1999). We examined the feasibility of operationalizing guidelines and monitoring the use of medication treatment guidelines in a CMHC. Specifically, we reviewed three national guidelines and algorithms for schizophrenia (Lehman and Steinwachs, 1998a; The Expert Consensus Guideline Series, 1999; Chiles, Miller, Crismon Rush, Krasnoff, and Shon, 1999) and identified a set of maintenance treatment recommendations that could be assessed using data available in a cross-sectional, retrospective chart review. We then examined the extent to which current pharmacological treatment practice at the CMHC conformed to those recommendations.

\section{METHODS}

The study was conducted in a small urban CMHC in the Midwest affiliated with a large university academic medical center. Three hundred and nine patients aged 18 years of age and older and diagnosed with schizophrenia or schizoaffective disorder, selected randomly from the center's data bank, comprised the study sample. A trained research assistant, using a data abstraction form designed for the study, conducted medical record reviews. Data were collected on patient demographics, diagnosis, duration of illness, symptoms and side effects, and medication.

We reviewed the recommendations for maintenance pharmacological treatment of schizophrenia in three sets of national guidelines: the Patient Outcomes Research Team (PORT) for schizophrenia (Lehman and Steinwachs, 1998a), the Texas Medication 
Algorithm Project (TMAP; Chiles, Miller, Crismon, Rush, Krasnoff, and Shon 1999), and the Expert Consensus Guideline series (1999). We identified five recommendations that could be evaluated using a cross-sectional medical chart review, and for which the required information was available in at least $50 \%$ of the medical records. Each recommendation was translated into a conformance criterion, which was then applied to current practices to estimate a conformance rate (Agency for Health Care Policy and Research, 1995).

The first guideline suggests that persons with schizophrenia or schizoaffective disorder who experience acute symptom relief with an antipsychotic should continue to receive this medication for at least one year. Given the constraints of the available data, the compliance criterion we assessed was whether individuals with these diagnoses were being prescribed an antipsychotic medication.

A second guideline recommends that the maintenance dosage of antipsychotic medication be in the range of 300-600 mg chlorpromazine (CPZ) equivalents per day. Our crosssectional data could not be used to determine whether a patient's current medication was prescribed for acute or maintenance treatment. However, since more than $80 \%$ of the sample had been ill for at least 10 years, we assumed that most patients were on maintenance doses and created a conformance criterion that assessed whether the antipsychotic dosages prescribed for patients were between 300-600 mg CPZ equivalents/day. Only those patients prescribed one oral antipsychotic medication were included in the assessment.

The PORT study advises that patients with severe TD be offered a trial of clozapine, while Consensus guidelines recommend that patients with mild TD be switched to risperidone or vitamin $\mathrm{E}$ be added to their current antipsychotic treatment. To operationalize these recommendations, we defined severe TD as an AIMS score of $\geq 3$, indicating that the patient had minimal abnormal movements in at least three parts of the body or mild to moderate movements in at least one. Mild TD was defined as an AIMS score of one or two.

A fourth guideline recommends that persons who experience persistent, clinically significant positive symptoms despite adequate antipsychotic therapy receive a trial of two or more antipsychotics, adjunctive pharmacotherapy, or clozapine. Since our crosssectional methodology provided no information on the use, duration, or outcome of previous medication trials, we drafted a conformance criterion that examined whether patients with positive symptoms noted in their medical record were receiving one or more of the above-recommended treatments.

The PORT guidelines recommend an "ongoing assessment" of the effectiveness/need for maintenance antipsychotic therapy and anti-parkinsonian agents. The Consensus guidelines specifically recommended monthly clinic visits for compliant, asymptomatic patients and evaluations for TD at a minimum of twice a year. The conformance criterion we used was whether patients had a clinic visit within four weeks of the chart review, and an AIMS test within three months (reflecting the CMHC's own higher standard for TD assessments).

\section{RESULTS}

\section{Sample Characteristics}

Of the 309 subjects, $43 \%$ were female; $64 \%$ were Caucasian and $30 \%$ were African-American. Mean (SD) age was 46.4 (11.9) years (range: 
20-86 years). Eighty-one percent were diagnosed with schizophrenia; $19 \%$ with schizoaffective disorder. Mean (SD) duration of illness was 21.1 (11.4) years. Mean (SD) score on the Global Assessment of Functioning (GAF) scale was 49.3 (9.5).

\section{Medical Record Review}

Much of the clinical information needed to assess guideline conformance was not documented in the medical record. For example, while the presence or absence of positive symptoms was noted in three-quarters of the charts, the presence or absence of negative symptoms was documented in only $6 \%$ (18). This lack of information limited the number of guidelines that could be evaluated.

\section{Conformance to Guidelines}

Conformance rates for the five criteria are listed below:

Criterion 1: 299 (96.8\%) of this sample were being treated with an antipsychotic.

Criterion 2: Of the 179 prescriptions for monotherapy antipsychotic treatment, $43 \%$ were within the recommended dose range of 300$600 \mathrm{mg}$ CPZ equivalents/day; $26 \%$ were below, and $32 \%$ were above (Table 1).

Criterion 3: Of the forty-six patients identified with severe TD, 63.0\% were being treated with an atypical antipsychotic ( $23.9 \%$ with clozapine). Just over one-third (36.4\%) of those with mild TD received either an atypical antipsychotic and/or vitamin $\mathrm{E}$.

Criterion 4: Information regarding the presence or absence of positive symptoms was noted in the medical charts for 224 subjects; positive symptoms were present in 83 and absent in 141. Of those with positive symptoms, $70(84.3 \%)$ were receiving at least one of the recommended treatments.

Criterion 5: Just over one-half (54.4\%) of the subjects had a clinic visit within four weeks of data collection; similarly just over half (54.0\%) had an AIMS test within the past three months.

\section{DISCUSSION}

Nearly all patients (97\%) in our study were prescribed an antipsychotic medication. In an evaluation of the PORT guidelines, Lehman and Stein- 
Mona Goldman, Ph.D., et al.

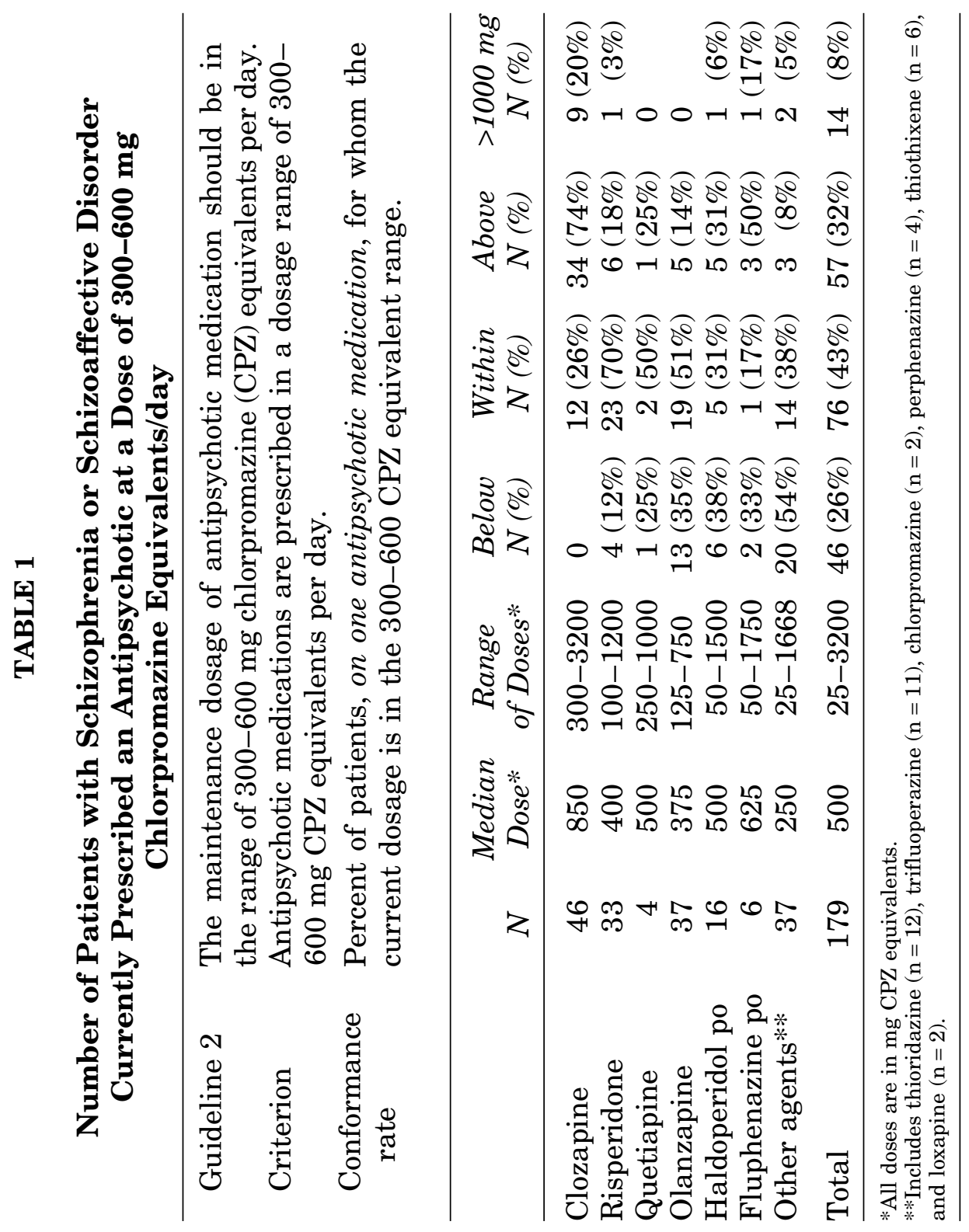


wach (1998b) reported that $92 \%$ of outpatients were treated with an antipsychotic. Using administrative data from a Veterans Affairs health center, Chen, et al. (2000) found a 77\% prescribing rate, perhaps reflecting patient, rather than physician, adherence rates, since only prescriptions filled were measured and not prescriptions written.

Only $43 \%$ of patients in our sample received the recommended antipsychotic maintenance dose of 300-600 mg CPZ equivalents, while $26 \%$ were below treatment guidelines and $32 \%$ above. Similar results were reported in the PORT evaluation (Lehman and Steinwachs, 1998b) where $29.1 \%$ of patients were in the recommended range; $31.9 \%$ above and $39.1 \%$ below. In the VA clinic study Chen et al. (2000) found 53\% of patients were within a range of $300-1000 \mathrm{mg} \mathrm{CPZ}$ equivalents, with $42 \%$ below $300 \mathrm{CPZ}$ equivalents and $5 \%$ above $1000 \mathrm{mg}$. CPZ equivalents. (Similarly, 8\% of our patients were receiving antipsychotic doses above $1000 \mathrm{mg} \mathrm{CPZ}$ equivalents; 9 of these 14 patients were on clozapine.)

Young, et al. (1998), using a combined patient interview/chart review, reported that $79 \%$ of patients in two public clinics received inappropriate management of medication side effects (akathisia, parkinsonism, and TD). Of the forty-six CMH patients who met our criteria for severe TD (AIMS $\geq 3$ ) nearly two-thirds $(63 \%)$ were being treated with an atypical antipsychotic, $24 \%$ (11) with clozapine.

Eighty-three patients (26.9\%) in our study had a documented presence of residual positive psychotic symptoms. Seventy of those (84\%) received at least one of the recommended adjunctive treatments. We suspect that conformance rates for this criterion will increase as more studies addressing the use of adjunctive agents in refractory psychosis are published.

In our medical record review, we found that many charts lacked sufficient documentation of negative symptoms, medication side effects, and comorbid conditions to assess adherence to guidelines related to these key aspects of treatment. Similar deficiencies in the necessary documentation have been reported in a variety of treatment settings (Chen, Nadkarni, Levin, Miller, Tu, Ivanco, and Li, 2000; Dassori, Chiles, and Swenson-Britt, 2000; Lehman and Steinwachs, 1998b; Young, Sullivan, Burnam, and Brook, 1998). It is impossible to know whether information about symptomatology was missing because it was not present on evaluation or not documented. Young et al. (1998) suggest the latter: they found that half of the symptoms and less than fifteen percent of medication side effects revealed by patients in a structured interview were actually recorded in the medical record. 


\section{CONCLUSIONS}

A gap exists between national treatment recommendations for schizophrenia and current practice in community mental health centers. Guidelines are designed to improve quality of care by bridging that gap. However, current medical records do not provide adequate data for assessing adherence to guideline recommendations. Tools that allow for cross-sectional assessment of guideline conformance without excessive cost are needed. Standardized progress notes and computerized prescribing programs may be the first steps to more quickly and accurately assess health care delivery for the seriously and persistently mentally ill.

\section{REFERENCES}

Agency for Health Care Policy and Research (1995). Using Clinical Practice Guidelines to Evaluate Quality of Care. AHCPR Pub. No. 94-0046. Rockville, MD: Agency for Health Care Policy and Research, Public Health Service, U.S. Department of Health and Human Services.

American Psychiatric Association: Practice guideline for the treatment of patients with schizophrenia. (1997). American Journal of Psychiatry, 154(4 Suppl), 1-63.

Chen R.S., Nadkarni P.M., Levin F.L., Miller P.L., Tu Y.T., Ivanco T.L., Li Y. (2000). Using a computer database to monitor compliance with pharmacotherapeutic guidelines for schizophrenia. Psychiatric Services, 51, 791-794.

Chiles J.A., Miller A.L., Crismon M.L., Rush A.J., Krasnoff A.S., Shon S.S. (1999). The Texas Medication Algorithm Project: development and implementation of the schizophrenia algorithm. Psychiatric Services, 50, 69-74.

Dassori A.M., Chiles J.A., Swenson-Britt E. (2000). Implementing best-practice guidelines for schizophrenia in a public-sector institution. Psychiatric Services, 51, 972-979.

DeQuardo J.R., Tandon R. (1998). Do atypical antipsychotic medications favorably alter the longterm course of schizophrenia? Journal of Psychiatric Research, 32, 229-242.

Doherty J.P. (1999). Cost of treating mental illness from a managed care perspective. Journal of Clinical Psychiatry, 60(supp 13), 49-52.

Jibson M.D., Tandon R. (1998). New atypical antipsychotic medications. Journal of Psychiatric Research, 32, 215-228.

Lehman A.F., Steinwachs D.M. (1998a). At Issue: Translating research into practice: The Schizophrenia Patient Outcomes Research Team(PORT) treatment recommendations. Schizophrenia Bulletin, 24,1-10.

Lehman A.F., Steinwachs D.M. (1998b). Patterns of usual care for schizophrenia: initial results from The Schizophrenia Patient Outcomes Research Team (PORT) client survey. Schizophrenia Bulletin, 24,11-20.

Remington G., Shammi C.M., Sethna R., Lawrence R. (2001). Antipsychotic dosing patterns for schizophrenia in three treatment settings. Psychiatric Services, 52, 96-98.

The expert consensus guideline series. Treatment of schizophrenia 1999. (1999). Journal of Clinical Psychiatry, 60(suppl 11), 3-80.

Young A.S., Sullivan G., Burnam M.A., Brook R.H. (1998). Measuring the quality of outpatient treatment for schizophrenia. Archives of General Psychiatry, 55, 611-617. 